\title{
Vergleich von Hoshin mit anderen Steuerungsmethoden im Vertrieb
}

Über Hoshin Kanri hinaus gibt es andere Steuerungsmethoden für Vertrieb und Gesamtunternehmen. Dazu gehören im Wesentlichen $\mathrm{MbO} /$, Management by Objectives“, BSC/,Balanced Scorecard“ und OKR/,Objectives and Key Results“. Siehe zu MbO Drucker (1954), zur Balanced Scorecard Kaplan (1993) und zu OKR Grove (1983). Es gibt auch Ansätze, die eine Kombination von Hoshin Kanri mit der BSC/Balanced Scorecard oder mit OKR empfehlen, wie Witcher (2007) und DeBusk (2011) für die BSC und Kudernatsch (2019) zur Methode OKR.

Daneben existieren einfache Vertriebs-Dashboards und Sales-Cockpits auf Basis von ERP- oder CRM-Systemen oder selbst erstellte Lösungen mittels Microsoft Excel. „Viele Wege führen nach Rom“ bedeutet, es muss nicht zwingend eine bestimmte Lösung sein. Es heißt aber auch „Man sollte nicht mit Kanonen auf Spatzen schießen“: Überdimensionierte und zu komplexe Systeme und Methoden werden keine Akzeptanz finden und dadurch nutzlos sein. Der Aufwand für Einrichtung, Betrieb und Wartung muss zu Ihren Erwartungen, Zielen und den in Ihrem Unternehmen vorhandenen Möglichkeiten passen. Zwar kann Excel (fast) jeder und ein ERP-System nutzt Ihr Unternehmen vermutlich bereits ebenfalls. Ich empfehle, auf erprobte Methoden und Instrumente zurückzugreifen, die nicht von den Programmierfähigkeiten eines Excel-Experten abhängen: Geht der Experte, geht meist auch die Fähigkeit zur Reparatur und Weiterentwicklung der eingesetzten Lösung verloren. Zudem: Bei der Verwendung einer auch in anderen Unternehmen genutzten Methode können Sie sich mit diesen austauschen und aus deren Erfahrungen und Weiterentwicklungen lernen. Hoshin Kanri kann daher für Sie eine Methode sein, die ideal für Ihr Unternehmen ist. 